Musées, Patrimoine et Culture scientifiques et techniques

$168 \mid 2016$

novembre-décembre 2016

\title{
Les risques psychosociaux
}

\section{Alain Michel}

URL : http://journals.openedition.org/ocim/1721

DOI : 10.4000/ocim. 1721

ISSN : 2108-646X

\section{Éditeur}

OCIM

Édition imprimée

Date de publication : 1 novembre 2016

Pagination : 31-35

ISSN : 0994-1908

Référence électronique

Alain Michel, «Les risques psychosociaux », La Lettre de l'OCIM [En ligne], 168 | 2016, mis en ligne le 01 novembre 2017, consulté le 19 avril 2019. URL : http://journals.openedition.org/ocim/1721 ; DOI : $10.4000 /$ ocim. 1721

Ce document a été généré automatiquement le 19 avril 2019.

Tous droits réservés 


\section{Les risques psychosociaux}

\section{Alain Michel}

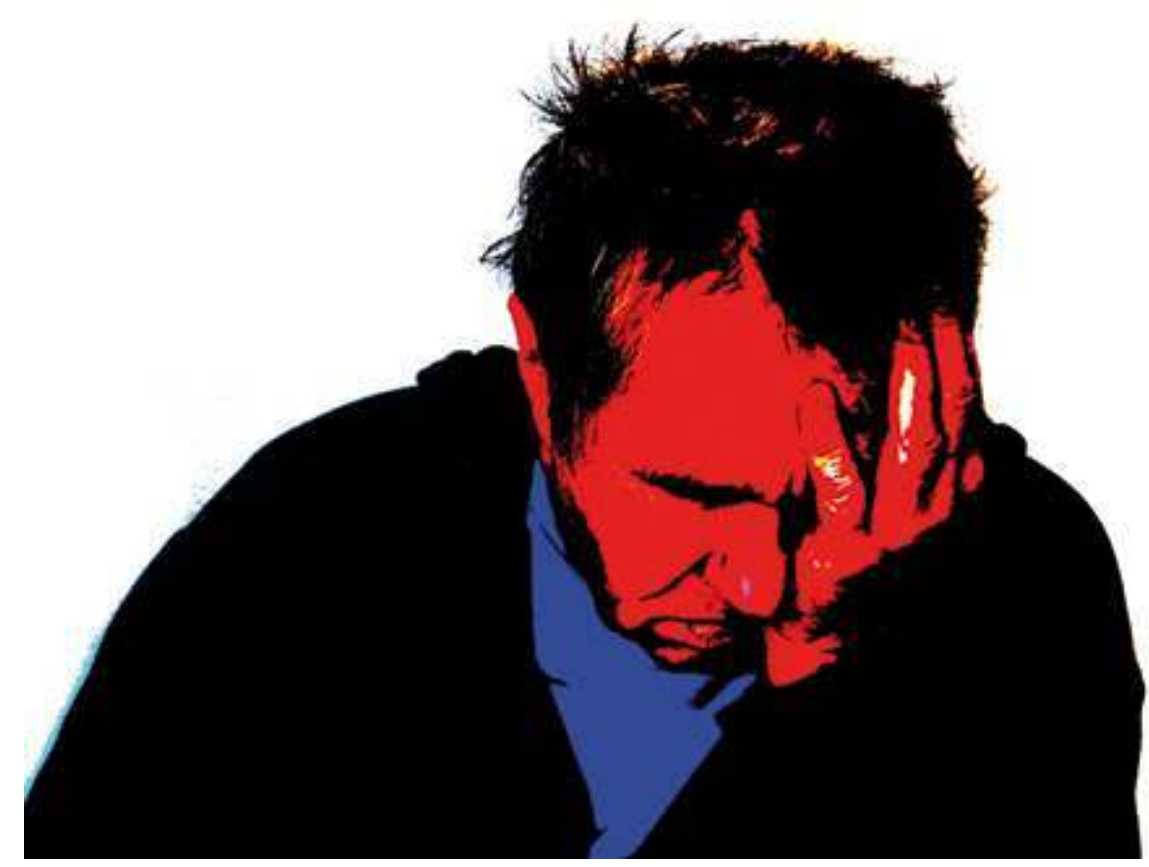

(c) IBUC

1 Dans les années 1970-1980, aux États-Unis, fut instauré un mode de management axé sur le harcèlement, le mobbing, que quelques-uns ont qualifié de psycho terreur. Il était sensé faire travailler plus les salariés en créant un climat délétère, il servait aussi à pousser des salariés ciblés à démissionner ou à faire disparaitre certains secteurs d'une entreprise au motif d'économie. Ce mode de management se généralisera dans les années 1980-1990 dans les pays développés mais, dès les années 1990, des économistes signalent que ce management n'a qu'une efficacité limitée, il fragilise les entreprises. Cependant, le mal est fait et dans les années 2000 certaines écoles supérieures et instituts de management 
continuent à enseigner ce type de gestion alors que l'on s'accorde par ailleurs à le traiter de fléau social.

2 Depuis les années 2000, économistes, juristes et multiples acteurs de la vie sociale ont montré que ce management n'était pas économiquement efficace, qu'il mène au déclin par la perte d'innovation et de créativité, à un absentéisme des salariés et à leur perte de motivation. Les coûts de santé de personnes subissant ces pressions ne cessent d'augmenter, les suicides liés à ce management dégradent l'image de marque des entreprises, s'ils sont nombreux, cela alerte les gouvernants. En France, la jurisprudence de la Cour de Cassation a reconnu ce mode de gestion comme étant un délit.

Aucun secteur d'activité n'est épargné et certains sont plus particulièrement affectés : financier et bancaire, administratif et gestion, commercial, sanitaire et social, industriel. Ce constat alarmant a poussé l'État à créer les commissions interprofessionnelles pour définir au mieux les risques psychosociaux (RPS), les prévenir afin d'éviter les atteintes à la santé physique et mentale des travailleurs : ces commissions ont abouti aux Accords Nationaux Interprofessionnels (ANI), dont le but est l'amélioration de la santé et de la sécurité au travail et donc de l'état économique et social du pays.

\section{Les effets sur la santé des RPS}

\section{Le stress au travail}

4 Le stress est un terme créé en 1936 par l'endocrinologue autrichien Hans Selye (1907-1982). Des stimulations externes fortes appelées stress (bruits, signaux visuels...) provoquent une réponse neuroendocrinienne quasi générale dans l'organisme qu'il appellera syndrome général d'adaptation. En effets immédiats visibles on aura : dilatation des pupilles, accélération du rythme cardiaque et constriction des vaisseaux sanguins (pâleur, extrémités froides), des douleurs abdominales par contractions de l'estomac et des intestins, des contractions musculaires. Les effets biologiques mesurables sont : une libération rapide d'hormones thyroïdienne et surtout d'adrénaline et de cortisol, qui provoquent l'accélération du rythme cardiaque, une poussée d'hypertension artérielle, une augmentation de la température d'où les sueurs froides. Il y a aussi une hyper sécrétion d'acide gastrique, une augmentation de la vigilance, avec éveil et perte de l'endormissement (préparation à l'attaque ou à la fuite). Dans un deuxième temps, l'adaptation provoque une hyperglycémie d'où sécrétion d'insuline, une augmentation du seuil de la douleur et une action anti inflammatoire (préparation à d'éventuelles atteintes physiques).

5 Un stress s'il est suffisamment fort, vu les interactions existantes entre le système nerveux et le système endocrinien (dosage cérébral très fin des taux d'hormones par le système limbique et leur régulation par l'axe hypothalamo-hypophysaire) sera mémorisé, selon les individus et leur vécu on verra des réponses différentes au même stress.

6 Dans les années 1950, le professeur Henri Laborit décrira la réponse oscillante post agressive : le taux élevé de cortisol post stress ne chute pas de façon linéaire, il évolue sur 21 jours selon une sinusoïde avec des haut et des bas (l'hypothalamus freine sa sécrétion, le taux devient trop bas, il en fait produire), un stress a des effets assez longs.

7 Des études plus récentes montrent que les récepteurs aux catécholamines (cortisol, corticostérone, adrénaline, noradrénaline...) du système limbique en cas de stress 
chronique se désensibilisent, la production des catécholamines n'est plus freinée, les surrénales s'épuisent d'où l'épuisement physique et la mort. Hans Selye avait constaté que le stress chronique entraînait le décès des rats qu'il étudiait.

8 Un stress aigu peut avoir des conséquences physiques et psychiques, notamment après une agression. Au plan physique, il y aura souvent un état de résistance ou d'adaptation en conséquence immédiate, suivi quelques heures après par une phase d'abattement ou de récupération. Les conséquences psychiques du fait de la mémorisation et du vécu pourront être importantes et durables, de la simple angoisse le matin en allant au travail, à des phobies et à l'impossibilité de retourner sur les lieux de travail, de l'insomnie passagère à l'insomnie rebelle, de l'état dépressif passager à la dépression grave et au suicide.

\section{Le stress chronique}

9 Le stress chronique est le plus pathogène. Une phase de décompensation s'installe plus ou moins rapidement si la personne ne trouve pas de réponse adaptée et si son entourage professionnel et familial ne la soutient pas; c'est le rôle des facteurs sociaux. C'est la phase d'épuisement, elle s'accompagne de quatre grands types de symptômes :

10 - des symptômes physiques : brûlures d'estomac, coliques, céphalées, anorexie ou boulimie, oppression thoracique, palpitations, essoufflement, sueurs, douleurs musculaires ou articulaire ;

11 - des symptômes émotionnels : angoisses, excitation ou abattement, tristesse, mal-être, sensibilité et nervosité accrues (crises de larmes, crises de nerfs);

12 - des troubles intellectuels : inhibition (difficulté à prendre des décisions, se met en retrait), perte d'initiative, troubles de la concentration (oublis, erreurs);

13 - des troubles du comportement : repli sur soi, diminution des activités sociales, risque d'addiction par le recours à des produits calmants et ou dopants (somnifères, anxiolytiques, antidépresseurs, café, tabac, alcool, drogues). 
Le système limbique interface entre le système endocrinien, le cerveau et la mémorisation(source : www.corpshumain.ca/Cerveau2.php)

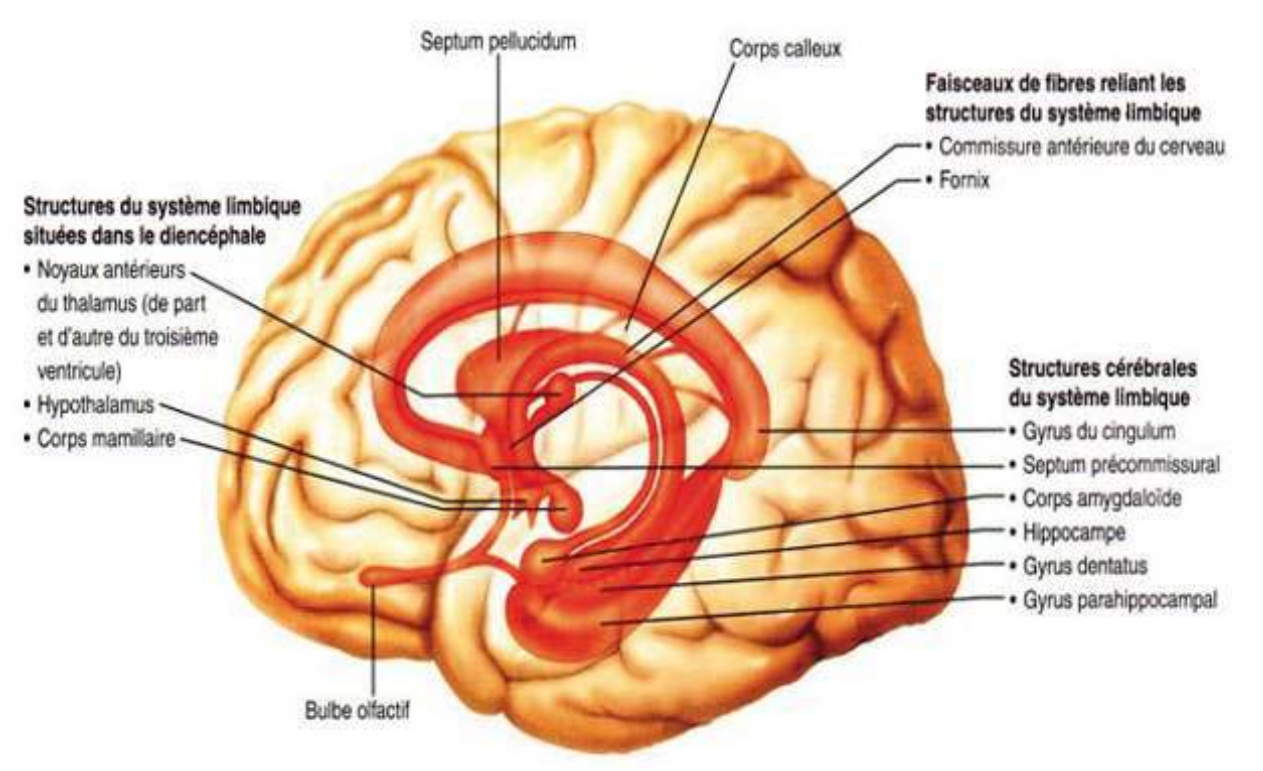

(C) DR

14 Les multiples enquêtes épidémiologiques menées depuis les années 1950 ont permis de mesurer les pathologies liées au stress :

15 - les pathologies cardiovasculaires : hypertension artérielle, augmentation du taux de cholestérol sanguin, atteinte des artères coronaires; ce phénomène est connu dès les années 1950 chez les cadres stressés mais cela affecte toutes les catégories professionnelles, préférentiellement les personnes au contact du public ;

16 - le syndrome métabolique: validé au début des années 2000, il était largement prévisible étant précurseur des pathologies cardiovasculaires ; l'hypersécrétion des catécholamines provoque une hypertension artérielle et une hyperglycémie qui amène une hypersécrétion d'insuline favorisant les crises de boulimies, un cercle vicieux s'installe obésité abdominale, résistance à l'insuline (pré diabète), désordres des lipides sanguins (triglycérides augmentés, cholestérol augmenté) ;

17 - les pathologies mentales : elles étaient attribuées à des fragilités individuelles, des études menées sur des cohortes importantes de divers publics (1996 sur des militaires pendant la guerre du Golfe, jusqu'à des études en 2004 sur l'addiction à l'alcool) prouvent que ces pathologies sont indépendantes des facteurs de personnalité, et qu'il existe une différence de réponse selon les sexes. Les femmes sont plus fragiles quand le soutien social est faible et les contraintes physiques fortes, les hommes plus sensibles au manque de latitude décisionnelle. Nous aurons ici les mêmes symptômes émotionnels, intellectuels et comportementaux constatés en phase de décompensation, auxquels s'ajoute une atteinte de la mémoire, par une stimulation de l'axe hypothalamohypophysaire-surrénal (axe HPA). L'hyper activité de l'axe HPA va perturber les rythmes nycthéméraux du cortisol, du sommeil, toute anomalie du sommeil crée un manque de récupération physique et psychique, la détresse psychologique ainsi induite fait le lit de la dépression, et des tentatives de suicides. On peut voir aussi des bouffées délirantes ou une paranoïa ; 
18 - les troubles musculosquelettiques (TMS) : des études canadiennes (1997), anglaises (2002) montrent que les RPS augmentent l'incidence des TMS, le stress provoque des contractures musculaires reflexes ;

19 - les baisses de l'immunité : des études épidémiologiques menées de 1985 à 2004 le prouvent, le cortisol étant anti inflammatoire, il provoque une baisse de l'immunité s'il agit longtemps, et l'on constate une fragilité aux infections et une augmentation des maladies auto-immunes.

Les effets biologiques du stress (source : www.ipubli.inserm.fr/bitstream/handle/10608/217/ Chapitre_13.html)

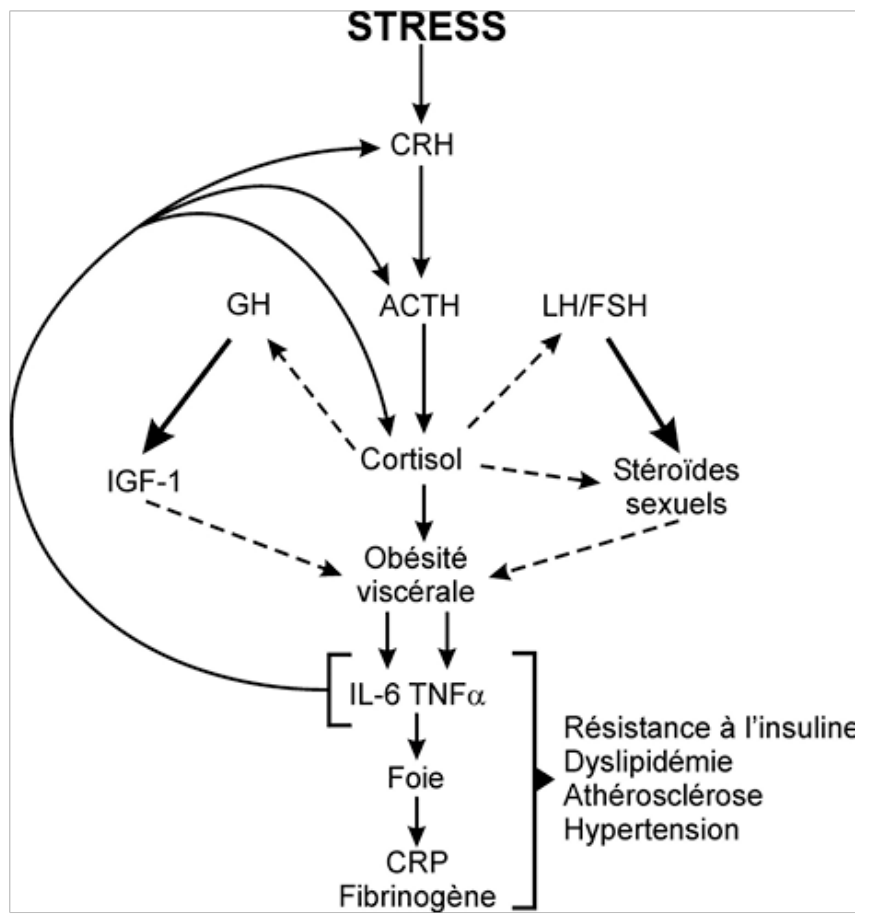

(C) DR

\section{Les effets socioéconomiques des RPS} facilement quantifiables (l'absentéisme, le turn-over des salariés et les indemnités de licenciement), d'autres le sont plus difficilement (perte de motivation, perte d'innovation, absence d'initiative, recours systématiques à des procédures rigides imposées, erreurs, oublis, mécontentement apparent, rumeurs entretenues au sein et hors de l'entreprise).

Les coûts de santé peuvent être mesurés. Les pathologies dues aux RPS ne sont pas prises en charge au titre des maladies professionnelles (MP), ceci a un effet négatif sur leur prévention dans certaines entreprises (absence de majoration des coûts annuels des AT/ MP). Les coûts de santé sont importants : états dépressifs amenant des arrêts maladies de trois ans et plus, épuisements professionnels et le burn out nécessitant des arrêts d'un an et plus, soins à vie et rentes d'invalidité. 

d'intérêt scientifique appelé "Évolution et Relation en Santé au Travail" (EVREST), dirigé par le docteur Ariane Leroyer de l'université Lille 2. Le sixième rapport (164 pages) est à l'échelon national, 980 médecins du travail ont rendu 24903 questionnaires individuels de salariés recueillis en 2012 et 2013. Des résultats rendus en 2014, il ressort que 23,5\% des salariés signalent subir des pressions psychologiques, $22,3 \%$ signalent de la fatigue et $2,9 \%$ se soignent ; $19,5 \%$ se plaignent d'anxiété et de nervosité et 3,5\% se soignent ; 18,4 $\%$ ont des troubles du sommeil et 3,6 \% se soignent ; 7,3 \% présentent une association de ces trois symptômes. De grandes entreprises cherchent à appréhender les RPS en recourant à ce questionnaire (banques, Airbus, SNCF, EDF...).

\section{Les causes des RPS} du travail), pas d'aide (isolement), supprimer ou ajouter des tâches ou des responsabilités sans avertir, laisser s'installer des rapports sociaux avec absence de savoir-faire (pas de salutations, critiques injustifiées, insultes);

27 - les conflits de valeur : ne pas avoir les moyens de faire un travail de qualité, conflit éthique ; ni de merci, couper la parole, siffler la personne, mépris, injures publiques, aucune communication verbale), bousculer, agressions physiques ;

31 - les techniques d'isolement du salarié : séparation du collectif de travail (changement d'horaire, de lieu), absence d'information (réunion, nouveau process), consignes de ne plus communiquer avec le salarié ;

32 - les techniques d'attaques du geste de travail :

33 . par des injonctions paradoxales : faire refaire une tâche déjà faite, corriger des erreurs inexistantes, donner une procédure pour la tâche à éffectuer, contester la procédure, déchirer un rapport demandé en urgence en le déclarant inutile, consignes confuses et contradictoires, faire venir en urgence la personne et ne rien lui faire faire ;

34 . par la déqualification du poste de travail : suppression de tâches et de responsabilité contractuelles ou découlant du poste de travail, suppressions du bureau, du téléphone, de l'ordinateur, du véhicule, des outils ; local de travail vidé ;

35 . par surcharge de travail : objectifs irréalisables comme ordonner un travail urgent 5 minutes avant la fin du temps de travail, demander une disponibilité de tous les moments. 
36 - les techniques de persécution : contrôle systématique (téléphone, ordinateur, courriers, casier, armoire, poubelle, sac à main, pauses), enregistrement des faits et des communications ;

37 - les techniques punitives : demander de tout justifier, notes de services pour tout, avertissement sans motif, lettres recommandées, congés refusés au dernier moment, heures supplémentaires ou indemnités non payées, horaires incohérents modifiés sans cesse.

L'icerberg de la connaissance

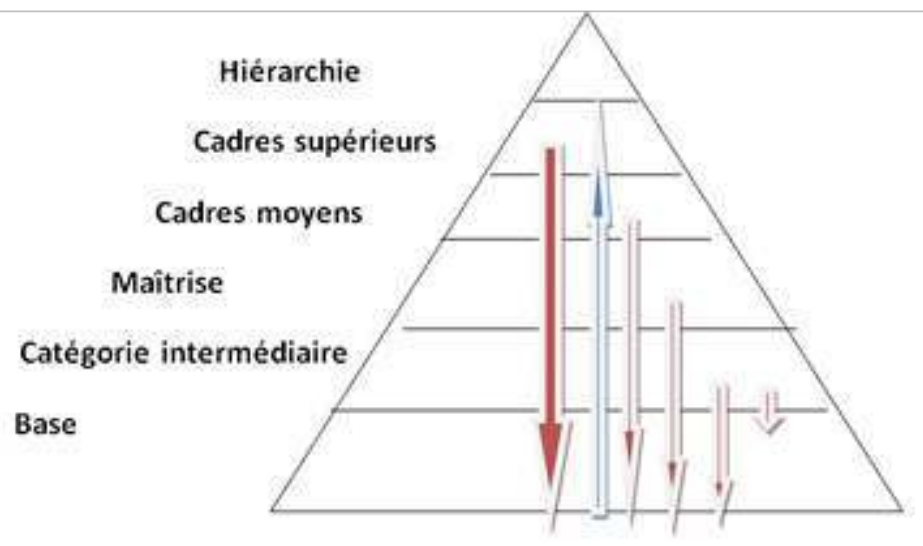

\section{L'iceberg de la connaissance}

La base reçoit des ordres de tous les étages,

Elle ne fait remonter que $4 \%$ d'informations à la hiérarchie

\section{La prévention des RPS}

\section{pour assurer la sécurité et protéger la santé physique et mentale des travailleurs". Cinq types de} mesures sont ainsi prévues:

L'article L4121-1 du Code du Travail précise que "l'employeur prend les mesures nécessaires - actions de prévention des risques professionnels et de pénibilité au travail ;

- actions de formation et d'information;

- mise en place d'une organisation et de moyens adaptés ;

- veiller à l'adaptation de ces mesures en fonction des changements ;

- planification de la prévention : la loi de 2012 dans l'alinéa 7 stipule que "l'employeur planifie la prévention en y intégrant dans un ensemble cohérent, la technique, l'organisation du travail, les relations sociales et l'influence des facteurs ambiants notamment les risques liés au harcèlement".

Le document unique d'évaluation des risques (DUER) (Loi du 31/12/991) a été rendu obligatoire le 8 novembre 2002. Toute entité de travail doit créer et réactualiser 
annuellement un DUER où il est mentionné les risques et leur importance (les RPS y figurent) et les mesures prises.

L'évaluation des risques favorisant les RPS (sept items de facteurs favorisant, cela n'est pas exhaustif) : l'organisation/management ; la communication interne; les techniques et process de travail ; les conditions de travail et l'environnement de travail (général et à chaque poste) ; l'état des relations dans l'établissement (hiérarchique et entre individus); les facteurs individuels (comportements) ; les facteurs externes (incivilités, intimidations, récriminations, agressions, vols...).

Les mesures correctives des RPS ne sont efficaces que si le climat de confiance est rétabli ; certains facteurs favorisant sont interdépendants :

47 - l'organisation et le management doivent être moins autoritaires, répressifs et occultes : quels sont les objectifs, sont-ils raisonnables donc atteignables, qui est qui, qui fait quoi ;

48 - une hiérarchie crédible sait trouver la bonne adéquation entre responsabilité et contrôle du travail : elle doit assurer un soutien adéquat aux personnes, aux équipes ou au contraire sanctionner, ou avertir quand cela est nécessaire ; les mesures, les décisions seront acceptées facilement ;

49 - la communication dans l'entreprise ne se réalise que si ces deux premiers points sont effectifs. Dans tout management autoritaire la haute hiérarchie ne connaît que $4 \%$ des réalités de la base (l'iceberg de la connaissance), les gens de la base subissent une cascade d'ordres, d'injonctions à ne pas commenter ou discuter, ils ne feront que peu remonter les informations, leurs difficultés ; cela crée le repli sur soi, les petits clans, les délations et les vengeances, il arrive que ceux qui travaillent soient sanctionnés. La communication n'est efficace que si la hiérarchie veut bien s'occuper de la réalité du terrain ;

50 - améliorer l'environnement et les conditions de travail, est bénéfique au climat social (moins de difficultés et de risques, sensation d'être écouté) ;

51 - clarifier le rôle de chacun, mais aussi laisser à chacun la possibilité d'évoluer, chacun pouvant trouver sa place ;

52 - chercher l'équité en cas de conflits entre acteurs de l'entreprise ou en cas de signalement de comportements individuels particuliers, en recoupant plusieurs avis pour connaître les faits, leurs motifs réels et aussi éviter qu'une personne ne soit la "tête de turc" désignée.

53 - concernant les facteurs externes :

54 . les subalternes subissent des incivilités et des agressions verbales voire physiques (caissières, agents d'accueil, secrétaires, agents de sécurité), il faut venir les soutenir, faire nombre, alerter les secours si besoin. Le supérieur hiérarchique doit intervenir, beaucoup d'individus n'auront plus le même courage devant lui ;

55 . contre les agressions par des malfaiteurs ou terroristes armés, toutes les mesures techniques et les procédures sont à mettre en œuvre et à faire évoluer. Cependant rien n'arrête totalement ces individus, il faut anticiper, faire des exercices, savoir comment se comporter, alerter discrètement, savoir où se réfugier... Il faut aussi prévoir le soutien psychologique post-agression. 


\section{Conclusion}

Les risques psychosociaux posent de multiples problèmes, ils peuvent vite empoisonner le climat social d'un établissement créant des dégâts importants chez les salariés. Nul n'en est totalement protégé (changement rapide du climat social ou managérial), et nul n'est à l'abri d'être catalogué de pourvoyeur de stress ou pire encore. Les éviter demande une bonne dose de philosophie, de psychologie et de savoir faire en matière de relations humaines, malheureusement cela ne suffit pas toujours.

\section{Quelques définitions} la santé mentale et physique des personnes au travail. Les RPS englobent le stress au travail qui peut amener à la souffrance au travail jusqu'à l'épuisement au travail (burn out), les diverses formes de harcèlements au travail (moral, sexuel et discriminations) et les incivilités allant jusqu'à l'agression qu'elles soient internes à l'entreprise ou externe à celle-ci.

Accords Nationaux Interprofessionnels (ANI), ont défini le stress au travail le 8 juillet 2008, le harcèlement et la violence au travail le 26 mars 2010. L'INRS (Institut National de Recherche et de Sécurité) décrit dans sa brochure ED 6012 le harcèlement. La Loi du 6 août 2012 précise le harcèlement sexuel et certaines formes de discriminations.

Il est nécessaire de connaître la législation, l'État tend à abandonner la mutualisation du risque et à augmenter la pression judicaire.

\section{Définition du stress au travail (selon l'ANI)}

60 "État survenant lorsqu'il y a déséquilibre entre la perception qu'une personne a des contraintes que lui impose son environnement professionnel et la perception qu'elle a de ses propres ressources pour y faire face".

\section{Définition du harcèlement}

61 1/ Le harcèlement moral : définitions identiques dans le Code pénal (CP) article L 222-33-2 et le Code du Travail (CT) article L 1152-1 : "Aucun salarié ne doit subir les agissements répétés de harcèlement moral qui ont pour objet ou pour effet une dégradation de ses conditions de travail susceptibles de porter atteinte à ses droits et à sa dignité, d'altérer sa santé physique ou mentale ou de compromettre son avenir professionnel" ;

2/ Le harcèlement sexuel a été redéfini en 2012 dans le CP article L 222-33 et repris dans le CT article L 1153-1

"Aucune personne ne doit subir des faits :

64 
65 répétée, exercée dans le but réel ou apparent d'obtenir un acte de nature sexuelle, que celui-ci soit recherché au profit de l'auteur ou au profit d'un tiers".

Le Code pénal retient cinq circonstances aggravantes.

3/ La discrimination liée au harcèlement est redéfinie dans le CT articles L 1152-2 (harcèlement moral) et article L 1153-1 (harcèlement sexuel), dans le CP article L 225-1-1 (harcèlement sexuel).

L'interdiction de discrimination à l'égard des victimes et des témoins est étendue à l'ensemble des personnes en formation ou en stage dans l'entreprise.

69 Le Code pénal retient deux cas de discrimination liée au harcèlement sexuel. Le Code $d u$ Travail mentionne ces deux cas, il mentionne d'autres cas applicables dans la discrimination liée au harcèlement sexuel (tout les cas sont applicables dans la discrimination liée au harcèlement moral).

70 4/ La discrimination en raison de l'identité sexuelle, nouvelle rédaction de l'article L 1132-1 du CT.

\section{RÉSUMÉS}

Les risques psychosociaux sont une composante des risques professionnels. Ils peuvent être présents partout, quels que soient la taille de l'entreprise et le secteur d'activité, et ils n'épargnent personne, les dirigeants comme les salariés pouvant y être exposés : l'auteur s'attache à en décrire les mécanismes et les effets puis présente les différents modes de prévention.

\section{INDEX}

Mots-clés : risques psychosociaux

\section{AUTEUR}

\section{ALAIN MICHEL}

médecin du travail

c2a.michel@wanadoo.fr 